\title{
Gender differences in determinants of weight-control behaviours among adolescents in Beirut
}

\author{
Mona N Kanaan ${ }^{1, *}$ and Rema A Afifi ${ }^{2}$ \\ 'Department of Health Sciences, Seebohm Rowntree Building (Area 3), University of York, Heslington, York \\ YO10 5DD, UK: ${ }^{2}$ Department of Health Behavior and Education, Faculty of Health Sciences, The American \\ University of Beirut, Beirut, Lebanon
}

Submitted 11 August 2008: Accepted 18 December 2008: First published online 26 February 2009

\begin{abstract}
Objective: To investigate the association between self-esteem, physical activity, engaging in risky behaviours, social and cultural capital, attitudes towards thinness, parental and personal characteristics with weight-control behaviours among adolescents in Beirut, Lebanon.

Design: A community-based cross-sectional study. A stratified cluster sampling design was used and information gathered by means of a questionnaire. Associations were investigated using multinomial logistic regression, accounting for the survey design. Odds ratios were calculated for trying to lose weight, or trying to gain weight, compared with no weight-control behaviour.

Setting: The study was conducted in three underprivileged urban areas of Beirut in 2003.

Subjects: The sample size was 1294 adolescents aged 13-19 years.

Results: Determinants of weight-control behaviour among girls included their attitudes towards thinness $\left(\mathrm{OR}_{\mathrm{loss}}=4 \cdot 29, \mathrm{OR}_{\text {gain }}=0 \cdot 38\right)$, dissatisfaction with weight $\left(\mathrm{OR}_{\text {loss }}=10 \cdot 9, \mathrm{OR}_{\text {gain }}=9 \cdot 63\right)$, engaging in physical activity $\left(\mathrm{OR}_{\text {loss }}=2 \cdot 22\right)$, smoking $\left(\mathrm{OR}_{\mathrm{loss}} \approx 3\right)$ and the mother working $\left(\mathrm{OR}_{\text {loss }}=2 \cdot 77\right)$. Determinants of weight-control behaviour in boys included their attitudes towards thinness $\left(\mathrm{OR}_{\text {loss }}=14 \cdot 7, \mathrm{OR}_{\text {gain }}=\right.$ $0 \cdot 35)$, dissatisfaction with weight $\left(\mathrm{OR}_{\text {loss }}=15 \cdot 6, \mathrm{OR}_{\text {gain }}=17 \cdot 7\right)$, being involved in a cultural activity $\left(\mathrm{OR}_{\text {gain }}=2 \cdot 51\right)$, engaging in a fight $\left(\mathrm{OR}_{\text {loss }}=5 \cdot 25, \mathrm{OR}_{\text {gain }}=1 \cdot 64\right)$ and engaging in physical activity $\left(\mathrm{OR}_{\mathrm{loss}}=2 \cdot 56, \mathrm{OR}_{\text {gain }}=2 \cdot 17\right)$.

Conclusions: Attitudes towards thinness, weight dissatisfaction and physical activity are common determinants for weight-control among boys and girls, although to varying degrees of influence. Self-esteem, social capital, cultural capital and parental characteristics were not significant predictors when accounting for the other variables.
\end{abstract}

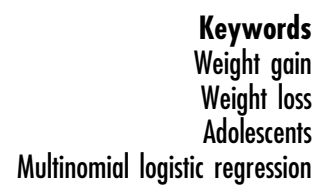

Keywords gain

escents

Multinomial logistic regression
Nutrition and weight-related issues are international concerns. The Millennium Development Goals include one goal specific to nutrition $^{(1)}$; and a recent fact sheet and reports discuss the epidemics of obesity ${ }^{(2,3)}$ on one the hand and malnutrition $^{(4,5)}$ on the other. The problems of both obesity and malnutrition have been documented in the Eastern Mediterranean region ${ }^{(3,6-9)}$. Interventions to reduce inappropriate nutrition and obesity need to be based on a thorough understanding of the determinants of such problems and associated behaviours. Several studies have looked at the prevalence of and determinants of weight control among adolescents. However, most of these studies were conducted in developed countries and very few studies were conducted in the Arab countries.

Weight-control behaviours include dieting (trying to lose weight), trying to maintain the same weight and trying to gain weight. Although each may have positive and/or negative consequences, dieting has been most frequently researched. The consequences of dieting can be severe ${ }^{(10)}$. In addition, several studies have shown that dieting habits acquired during adolescence tend to persevere into adulthood ${ }^{(11,12)}$. Dieting has also been identified as the most important predictor in the onset of adolescent new eating disorders ${ }^{(13)}$.

Recently, the need to understand the nature and interactions of social, psychological and physiological risk factors on body dissatisfaction and disordered eating has been highlighted ${ }^{(14)}$. Weight control and/or dieting behaviour has been found to be associated with several categories of variables including demographic, socio-economic, personal, socio-cultural, and other health behaviours.

Demographically, girls are more likely to be trying to lose weight than boys ${ }^{(15-19)}$ and boys are more likely to be trying to gain weight or do nothing about their weight than girls ${ }^{(17,19)}$. Socio-economically, previous study results are varied ${ }^{(15-17)}$ 
At the personal level, dieting frequency or unhealthful weight-control practices have been shown to be positively related to disordered eating and psychological problems such as body dissatisfaction, depression, feelings of insecurity and low self-esteem ${ }^{(10,20-25)}$. Sociocultural agents such as media, family and peers have been shown to influence adolescent girls' body dissatisfaction and dietary restraint ${ }^{(10,26,27)}$.

Related to other health risk behaviours, positive associations have been found between weight-control behaviours and behavioural risk factors among adolescents ${ }^{(18,19,24,28-30)}$. The association between smoking and dieting is well established, especially among girls ${ }^{(18,31,32)}$.

Several studies have looked at weight-related issues among high-school or university students in Lebanon ${ }^{(7,33-35)}$. In general, the results of the research in Lebanon are supportive of the international literature on determinants.

In order to advance understanding of the determinants of weight-control behaviour among young persons in Lebanon, the present study investigated the association between attitudes towards thinness, self-esteem, risky behaviours, social capital and parental characteristics and weight-control behaviours among girls and boys in three underprivileged urban areas of Beirut, Lebanon. Identifying the characteristics of such behaviours will enable health professionals to target those at risk with the appropriate interventions.

\section{Methods and materials}

\section{Study design}

The data used in the present analysis come from Phase II of the urban health survey conducted in three impoverished urban communities of Beirut, Lebanon in 2003 by the Centre for Research on Population and Health (CRPH) at the Faculty of Health Sciences of the American University of Beirut, Lebanon. The sample was based on stratified clustering by the three communities: Naba'a, Hay El-Sollom and Burj El-Barajneh. All adolescents who were between the age of 13 and 19 years inclusively were identified from households that were sampled in Phase I. Of the 2799 households sampled in Phase I, only 783 households, identified from seventy-one clusters, had eligible participants resulting in a sample size of 1526 adolescents. Of these, 9.6\% refused to participate and a further $4 \cdot 6 \%$ were lost to follow-up. Thus the final sample size of adolescents was 1294 , which is an $84.8 \%$ response rate. All questionnaires were conducted in Arabic.

\section{Materials}

In what follows, we give a brief description of the questions used in our analysis. The variables are grouped into six distinct blocks of variables representing distinct components that might bear influence on the weight-control behaviour of an adolescent. Our response variable is trying to gain weight, trying to lose weight, trying to stay the same weight or not trying to change weight.

\section{Block 1: Attitudes towards thinness and weight satisfaction}

Attitudes toward thinness were assessed by the following questions: (i) 'Do you think that the thinner you are, the better you look?' (ii) 'In your opinion, are girls pretty whatever their weight is?' (iii) 'In your opinion, do girls have to be thin or slim to be pretty?' (iv) 'Do you care if your friends are fat/thin?' (v) 'How satisfied are you with your weight?

\section{Block 2: Self-esteem}

Self-esteem questions included whether they were satisfied with themselves; whether they could achieve like others; and whether they felt like losers.

\section{Block 3: Personal characteristics}

Personal characteristics included engaging in cultural activities (such as participation in theatrical/musical/ dance acts or going to book/art exhibitions) and religiosity. Religiosity was assessed by whether they considered themselves religious and to what extent their religious beliefs affected their daily life.

\section{Block 4: Risky behaviours}

Risky behaviours included smoking cigarettes, smoking argileb (also known as hookah or water-pipe), engaging in fights within the past three months and absence of physical activity. Physical activity was defined in two ways: following Lowry et $a l^{(36)}$ and following Sibai et $a l^{(7)}$. According to the first method ${ }^{(36)}$, physical activity was divided into vigorous (e.g. playing football), moderate (e.g. walking), or muscle strengthening (e.g. weight lifting); while according to the second ${ }^{(7)}$, physical activity was considered to be present if the respondent engaged in any physical activity on three or more days per week.

\section{Block 5: Social capital}

Social capital was assessed by whether the adolescents: belonged to a union or a social/neighbourhood/cultural/ athletic club; engaged in social activities, such as attending a general meeting, writing to a newspaper or taking part in a demonstration or a strike within the last six months; knew/trusted people in the neighbourhood; and were satisfied with the number of family members/friends living nearby. In addition, we queried the relationship of the person the adolescents turn to if in urgent need of ride, in need to borrow 15 000LL (equivalent to \$US 10) or if they have a personal problem.

\section{Block 6: Parental characteristics}

Parental characteristics were assessed as years of education and occupation of both parents. 


\section{Statistical analysis}

To assess the effect of some of the perceived factors, multivariate multinomial-logistic regression modelling was used while accounting for the design effect. We analysed the data in two stages. First, for each block of variables, we built a model - termed as Model I - using a stepwise backward elimination. Therefore, we performed six different regressions. For each regression, the initial step included all variables listed in the block and the following variables: education, age, pocket money, general health and health services utilization, which were retained throughout the selection process. For the retention of variables in the stepwise regression a $P$ value of $0 \cdot 1$ was used. Second, Model II was built by including all of the variables retained in the final step of the six regressions. For this model, a $P$ value less than 0.05 was considered to be statistically significant.

All analyses were stratified by gender. Merging of parents' characteristics that were not available in the adolescent file and re-coding were done using the $\mathrm{R}$ programming language and environment ( $\mathrm{R}$ Foundation for Statistical Computing, Vienna, Austria). All statistical analyses were done using the STATA statistical software package version 9 (StataCorp LP, College Station, TX, USA). Odds ratio estimates are reported together with 95\% confidence intervals.

\section{Results}

A total of 1294 unmarried adolescents, 617 (population size 743) girls and 677 (population size 804) boys, aged 13-19 years, answered the survey. Fifty-two per cent were boys. Overall, $11 \%$ of the sample was trying to lose weight, $9 \%$ was trying to gain weight, $3 \%$ was trying to stay the same weight and the remaining was doing nothing about their weight. Since the percentage trying to stay the same weight was so low, we combined this group with those doing nothing about their weight in order to avoid any unstable estimates that might result from the low percentage. Henceforth, we refer to this group as those who were doing nothing about their weight and abbreviate it as DONOWT. Furthermore, we refer to those who were trying to lose or gain weight as LOSEWT and GAINWT, respectively.

Among girls, 75\% were not doing anything particular about their weight inclusive of those who were trying to stay the same weight (5\%), 20\% were trying to lose weight and the other $5 \%$ were trying to gain weight. Similarly, among boys, the majority were doing nothing particular about their weight (82\%). However, in contrast to girls, those trying to lose weight were only $5 \%$ as compared with $13 \%$ trying to gain weight.

The average age for girls was 15.6 years and for boys 15.9 years. Approximately $40 \%$ of girls had less than 8 years of education, compared with $47 \%$ of boys. About $28 \%$ of boys did not receive any pocket money compared with $10 \%$ of girls. The distribution of these demographic variables was homogeneous across the three weightcontrol groups.

Three-quarters of the adolescents reported that they enjoyed good to very good health. For both genders, the proportion of those who perceived themselves as having poor/very poor/acceptable health was highest among GAINWT. Approximately half of the sample had seen a medical doctor within the last year. Furthermore, the proportion of those who perceived their health as worse than others ( $8 \%$ of girls and $10 \%$ of boys) was statistically significantly higher among GAINWT compared with the other two groups for both genders.

Table 1 gives the general characteristics of the sample and the crude odds ratios together with corresponding 95\% CI. Table 2 gives the estimates of the adjusted odds ratios under Models I and II. The reference group for the multinomial logistic regression models is DONOWT. In the following, we comment on the main findings of our study.

\section{Block 1: Attitude towards thinness and weight satisfaction}

Overall, $18 \%$ of boys and $40 \%$ of girls reported that one looked better the thinner one was; $37 \%$ of boys and $44 \%$ of girls stated that girls look beautiful regardless of their weight; and $18 \%$ of boys and girls reported that girls need to be thin to look beautiful. In addition, $77 \%$ of boys and $69 \%$ of girls were satisfied with their weight. The majority of boys and girls, approximately $96 \%$, stated that they did not care if their friends were fat or thin. These two variables were dropped in further analysis to avoid unstable estimates due to sparseness.

Among girls, LOSEWT were $4 \cdot 29$ (95\% CI 2·35, 7·82) times more likely to say that one looked better the thinner one was compared with DONOWT; in contrast, GAINWT were $0.38(95 \%$ CI $0 \cdot 15,0 \cdot 95)$ times less likely to say so. LOSEWT and GAINWT were approximately ten times more likely to be not satisfied with their weight compared with DONOWT. In addition, LOSEWT were 1.67 (95\% CI $1 \cdot 09,2 \cdot 55)$ times more likely to report that only thin girls look beautiful compared with DONOWT. No statistical difference was detected among the groups when asked if girls look beautiful regardless of their weight (see Table 1, crude estimates).

Adjusting for demographic and health-related variables, the variables retained in the final step were thinner is better and satisfied with weight. Under Model I, the effect of satisfaction with weight decreased to $\mathrm{OR}=8.47$ $(95 \%$ CI $4.59,15 \cdot 6)$ in LOSEWT and increased to OR $=$ $17.9(95 \%$ CI 8.06, 9.7) in GAINWT. One the other hand, the effect of thinner is better decreased for LOSEWT to $\mathrm{OR}=2 \cdot 04(95 \% \mathrm{CI} 1 \cdot 06,3 \cdot 94)$ and increased its protectiveness for GAINWT to OR $=0 \cdot 15(95 \%$ CI $0 \cdot 05,0 \cdot 45)$ (see Table 2, Model I estimates).

For boys, the crude estimates for GAINWT were similar to those of girls except for satisfaction with weight which 
Table 1 General characteristics of the sample stratified by gender and weight-control measure: adolescents aged 13-19-years from three underprivileged urban areas of Beirut, Lebanon, 2003. The $n(\%)$ are the observed counts and the weighted percentages. The crude odds ratios are presented with $95 \%$ confidence interval, with significant odds with respect to the DONOWT group indicated by italic font

\begin{tabular}{|c|c|c|c|c|c|c|}
\hline \multirow[b]{2}{*}{ Variable } & \multicolumn{3}{|c|}{ Girls } & \multicolumn{3}{|c|}{ Boys } \\
\hline & GAINWT & DONOWT & LOSEWT & GAINWT & DONOWT & LOSEWT \\
\hline Unweighted $n$ & 33 & 465 & 119 & 86 & 560 & 31 \\
\hline Weighted $n$ & 43 & 559 & 142 & 101 & 667 & 37 \\
\hline \multicolumn{7}{|l|}{ Block 1: Attitudes towards thinness and weight satisfaction } \\
\hline Thinner is better (no = ref; yes), $n(\%)$ & $6(18)$ & $171(37)$ & $84(72)$ & $6(7)$ & $95(17)$ & $23(75)$ \\
\hline OR $(95 \% \mathrm{Cl})$ & $0.38(0.15,0.95)$ & 1.00 & $4 \cdot 29(2 \cdot 35,7 \cdot 82)$ & $0.35(0.14,0.86)$ & $1 \cdot 00$ & $14 \cdot 7(6 \cdot 08,35 \cdot 6)$ \\
\hline $\begin{array}{l}\text { Satisfied with weight (satisfied/very satisfied = ref; not/not } \\
\text { at all/no opinion), } n(\%)\end{array}$ & $22(68)$ & $85(18)$ & $85(71)$ & $62(72)$ & $74(13)$ & $21(70)$ \\
\hline OR $(95 \% \mathrm{Cl})$ & $9 \cdot 63(4 \cdot 79,19 \cdot 4)$ & 1.00 & $10 \cdot 9(6 \cdot 23,19 \cdot 2)$ & $17 \cdot 7(9 \cdot 78,32 \cdot 1)$ & $1 \cdot 00$ & $15 \cdot 6(7 \cdot 26,33 \cdot 3)$ \\
\hline Only thin girls are beautiful (no = ref; yes), $n(\%)$ & $7(20)$ & $76(16)$ & $29(25)$ & $24(28)$ & $99(17)$ & $8(26)$ \\
\hline OR $(95 \% \mathrm{Cl})$ & $1.25(0.51,3.07)$ & 1.00 & $1 \cdot 67(1 \cdot 09,2 \cdot 55)$ & $1 \cdot 91(1 \cdot 07,3 \cdot 41)$ & $1 \cdot 00$ & $1 \cdot 68(0 \cdot 70,4 \cdot 01)$ \\
\hline $\begin{array}{l}\text { Girls look beautiful regardless of their weight (no = ref; } \\
\text { yes), } n(\%)\end{array}$ & $10(34)$ & $212(46)$ & $50(42)$ & $25(30)$ & $219(39)$ & $4(12)$ \\
\hline OR $(95 \% \mathrm{Cl})$ & $0 \cdot 61(0 \cdot 23,1 \cdot 59)$ & $1 \cdot 00$ & $0 \cdot 86(0 \cdot 54,1 \cdot 39)$ & $0.69(0 \cdot 40,1 \cdot 18)$ & $1 \cdot 00$ & $0.22(0.07,0.63)$ \\
\hline \multicolumn{7}{|l|}{ Block 2: Self-esteem } \\
\hline $\begin{array}{l}\text { Satisfied with oneself (agree }=\text { ref; disagree/no opinion), } \\
n(\%)\end{array}$ & $3(8)$ & $36(8)$ & $21(17)$ & $12(14)$ & $52(9)$ & $7(22)$ \\
\hline OR $(95 \% \mathrm{Cl})$ & $1 \cdot 04(0 \cdot 28,3 \cdot 81)$ & 1.00 & $2 \cdot 37(1 \cdot 38,4 \cdot 06)$ & $1 \cdot 61(0 \cdot 78,3 \cdot 33)$ & 1.00 & $2 \cdot 92(1 \cdot 30,6 \cdot 57)$ \\
\hline $\begin{array}{l}\text { Can achieve like others (agree = ref; disagree/no opinion), } \\
n(\%)\end{array}$ & $14(42)$ & $180(40)$ & $33(28)$ & $35(43)$ & $202(38)$ & $4(13)$ \\
\hline OR $(95 \% \mathrm{Cl})$ & $1 \cdot 09(0 \cdot 50,2 \cdot 34)$ & $1 \cdot 00$ & $0.58(0.38,0.89)$ & $1 \cdot 24(0 \cdot 77,2 \cdot 02)$ & $1 \cdot 00$ & $0.25(0.09,0.74)$ \\
\hline Feel like a loser (disagree = ref; agree/no opinion), $n(\%)$ & $7(18)$ & $69(15)$ & $23(19)$ & $12(14)$ & $71(13)$ & $5(16)$ \\
\hline OR $(95 \% \mathrm{Cl})$ & $1 \cdot 21(0 \cdot 51,2 \cdot 88)$ & $1 \cdot 00$ & $1 \cdot 32(0 \cdot 79,2 \cdot 19)$ & $1 \cdot 05(0 \cdot 48,2 \cdot 29)$ & $1 \cdot 00$ & $1 \cdot 26(0 \cdot 43,3 \cdot 71)$ \\
\hline \multicolumn{7}{|l|}{ Block 3: Personal characteristics } \\
\hline Engaged in a cultural activity (no = ref; yes), $n(\%)$ & $25(73)$ & $319(69)$ & $94(80)$ & $72(84)$ & $374(68)$ & $27(87)$ \\
\hline OR $(95 \% \mathrm{Cl})$ & $1 \cdot 20(0.51,2 \cdot 84)$ & 1.00 & $1 \cdot 73(1 \cdot 14,2 \cdot 64)$ & $2 \cdot 51(1 \cdot 21,5 \cdot 19)$ & 1.00 & $3.15(0.98,10.09)$ \\
\hline $\begin{array}{l}\text { Degree of religiosity (half-half = ref; very religious/ } \\
\text { religious), } n(\%)\end{array}$ & $12(34)$ & $195(43)$ & $45(37)$ & $54(65)$ & $331(59)$ & $17(55)$ \\
\hline OR $(95 \% \mathrm{Cl})$ & $0 \cdot 71(0 \cdot 28,1 \cdot 80)$ & $1 \cdot 00$ & $0.83(0.51,1.35)$ & $1 \cdot 35(0 \cdot 76,2 \cdot 40)$ & $1 \cdot 00$ & $0 \cdot 76(0.34,1 \cdot 68)$ \\
\hline (half-half $=$ ref; not $/$ not at all religious), $n(\%)$ & $2(6)$ & $16(3)$ & $8(7)$ & $9(9)$ & $53(9)$ & $2(6)$ \\
\hline OR $(95 \% \mathrm{Cl})$ & $1.57(0 \cdot 39,6 \cdot 39)$ & $1 \cdot 00$ & $1 \cdot 81(0 \cdot 71,4 \cdot 62)$ & $1 \cdot 21(0 \cdot 54,2 \cdot 70)$ & 1.00 & $0.52(0 \cdot 11,2 \cdot 36)$ \\
\hline Religion affects daily life (a little = ref; a lot), $n(\%)$ & $9(32)$ & $174(39)$ & $43(36)$ & 27 (34) & $248(47)$ & $11(36)$ \\
\hline OR $(95 \% \mathrm{Cl})$ & $0.62(0 \cdot 20,1 \cdot 94)$ & 1.00 & $1 \cdot 07(0 \cdot 67,1 \cdot 72)$ & $0.55(0.28,1.08)$ & 1.00 & $0 \cdot 72(0 \cdot 29,1 \cdot 77)$ \\
\hline (a little = ref; not at all), $n(\%)$ & $4(11)$ & $76(17)$ & $29(25)$ & $26(30)$ & $138(26)$ & $10(34)$ \\
\hline OR $(95 \% \mathrm{Cl})$ & $0.48(0 \cdot 14,1 \cdot 65)$ & 1.00 & $1 \cdot 67(0 \cdot 88,3 \cdot 16)$ & $0.86(0.47,1.55)$ & $1 \cdot 00$ & $1 \cdot 20(0 \cdot 49,2 \cdot 96)$ \\
\hline \multicolumn{7}{|l|}{ Block 4: Risky behaviours } \\
\hline Ever tried cigarettes (never $=$ ref; ever), $n(\%)$ & $3(7)$ & $58(12)$ & $26(22)$ & $30(36)$ & $167(30)$ & $10(33)$ \\
\hline OR $(95 \% \mathrm{Cl})$ & $0.59(0 \cdot 17,2 \cdot 01)$ & 1.00 & $1.96(1 \cdot 16,3 \cdot 30)$ & $1 \cdot 33(0 \cdot 78,2 \cdot 26)$ & 1.00 & $1 \cdot 15(0 \cdot 48,2 \cdot 77)$ \\
\hline Ever tried argileh (never = ref; ever), $n(\%)$ & $10(27)$ & $175(38)$ & 78 (67) & $73(84)$ & $356(64)$ & $24(78)$ \\
\hline OR $(95 \% \mathrm{Cl})$ & $0.60(0.29,1.24)$ & 1.00 & $3.30(2 \cdot 09,5 \cdot 20)$ & $3.01(1 \cdot 47,6 \cdot 18)$ & 1.00 & $1.95(0.85,4.49)$ \\
\hline Violence in the past three months (no = ref; yes), $n(\%)$ & $1(2)$ & $21(4)$ & $7(5)$ & $22(27)$ & $101(18)$ & $17(54)$ \\
\hline OR $(95 \%$ Cl) & $0.56(0.09,3.37)$ & 1.00 & $1 \cdot 28(0 \cdot 48,3 \cdot 43)$ & $1 \cdot 64(1 \cdot 03,2 \cdot 60)$ & $1 \cdot 00$ & $5.25(2.51,11 \cdot 0)$ \\
\hline
\end{tabular}




\begin{tabular}{|c|c|c|c|c|c|c|}
\hline \multirow[b]{2}{*}{ Variable } & \multicolumn{3}{|c|}{ Girls } & \multicolumn{3}{|c|}{ Boys } \\
\hline & GAINWT & DONOWT & LOSEWT & GAINWT & DONOWT & LOSEWT \\
\hline Sibai's physical activity (yes $=$ ref; no), $n(\%)$ & $27(82)$ & $365(79)$ & $75(63)$ & $43(49)$ & $378(68)$ & $14(45)$ \\
\hline OR $(95 \% \mathrm{Cl})$ & $1 \cdot 17(0.45,3.06)$ & $1 \cdot 00$ & $0.45(0.28,0.70)$ & $0.46(0.30,0.72)$ & 1.00 & $0.39(0.17,0.89)$ \\
\hline Lowry's physical activity (yes = ref; no), $n(\%)$ & $31(95)$ & $411(89)$ & $89(74)$ & $54(62)$ & $404(72)$ & $16(51)$ \\
\hline OR $(95 \% \mathrm{Cl})$ & $2 \cdot 24(0.51,9.89)$ & $1 \cdot 00$ & $0.37(0.23,0.58)$ & $0.63(0.41,0.98)$ & $1 \cdot 00$ & $0.40(0.19,0.84)$ \\
\hline \multicolumn{7}{|l|}{ Block 5: Social support } \\
\hline Membership of a club (no = ref; yes), $n(\%)$ & $6(18)$ & $83(18)$ & $34(27)$ & $37(44)$ & $188(34)$ & $20(65)$ \\
\hline OR (95\% Cl) & $0.96(0.33,2 \cdot 83)$ & $1 \cdot 00$ & $1 \cdot 70(0 \cdot 99,2 \cdot 93)$ & $1 \cdot 51(0 \cdot 99,2 \cdot 31)$ & 1.00 & $3.51(1.59,7 \cdot 74)$ \\
\hline $\begin{array}{l}\text { For an emergency lift, ask (family = ref; friends/others), } \\
n(\%)\end{array}$ & $5(14)$ & $37(8)$ & $11(9)$ & $16(18)$ & $90(16)$ & $5(16)$ \\
\hline OR $(95 \% \mathrm{Cl})$ & $1 \cdot 89(0.64,5 \cdot 59)$ & $1 \cdot 00$ & $1 \cdot 18(0.55,2 \cdot 55)$ & $1 \cdot 34(0 \cdot 72,2 \cdot 51)$ & 1.00 & $1.29(0.49,3 \cdot 38)$ \\
\hline (family = ref; no one), $n(\%)$ & $1(2)$ & $22(5)$ & $8(8)$ & $13(16)$ & $48(8)$ & $7(23)$ \\
\hline OR $(95 \% \mathrm{Cl})$ & $0.48(0.06,3 \cdot 74)$ & $1 \cdot 00$ & $1 \cdot 60(0 \cdot 62,4 \cdot 13)$ & $2 \cdot 21(1 \cdot 03,4 \cdot 76)$ & $1 \cdot 00$ & $3 \cdot 60(1 \cdot 28,10 \cdot 13)$ \\
\hline $\begin{array}{l}\text { In need to borrow } 15000 \mathrm{LL} \text {, ask (family = ref; friends/ } \\
\text { others/no one), } n(\%)\end{array}$ & $4(11)$ & $27(6)$ & $12(11)$ & $16(18)$ & $59(10)$ & $7(22)$ \\
\hline OR $(95 \% \mathrm{Cl})$ & $2 \cdot 01(0 \cdot 66,6 \cdot 16)$ & $1 \cdot 00$ & $2 \cdot 13(0 \cdot 99,4 \cdot 62)$ & $1.96(1 \cdot 05,3 \cdot 65)$ & 1.00 & $2 \cdot 43(0.92,6 \cdot 40)$ \\
\hline $\begin{array}{l}\text { Personal problem, ask (family = ref; friends/others } / \text { no } \\
\text { one), } n(\%)\end{array}$ & $7(19)$ & $69(15)$ & $30(25)$ & $22(25)$ & $124(21)$ & $15(46)$ \\
\hline OR $(95 \% \mathrm{Cl})$ & $1 \cdot 31(0 \cdot 41,4 \cdot 15)$ & $1 \cdot 00$ & $1 \cdot 84(1 \cdot 11,3 \cdot 05)$ & $1 \cdot 25(0 \cdot 74,2 \cdot 12)$ & $1 \cdot 00$ & $3 \cdot 19(1 \cdot 43,7 \cdot 11)$ \\
\hline $\begin{array}{l}\text { Know people in the neighbourhood (few/very few = ref; } \\
\text { many/most), } n(\%)\end{array}$ & $21(60)$ & $191(42)$ & $66(55)$ & $61(72)$ & $329(60)$ & $19(62)$ \\
\hline OR $(95 \% \mathrm{Cl})$ & $2 \cdot 05(0 \cdot 82,5 \cdot 09)$ & $1 \cdot 00$ & $1 \cdot 64(1 \cdot 09,2 \cdot 47)$ & $1 \cdot 72(0.90,3 \cdot 28)$ & $1 \cdot 00$ & $1 \cdot 08(0 \cdot 50,2 \cdot 33)$ \\
\hline $\begin{array}{l}\text { Trust people in the neighbourhood (few/very few = ref; } \\
\text { many/most), } n(\%)\end{array}$ & $4(10)$ & $24(5)$ & $3(3)$ & $10(12)$ & $39(7)$ & $1(4)$ \\
\hline OR $(95 \% \mathrm{Cl})$ & $2 \cdot 15(0 \cdot 56,8 \cdot 29)$ & $1 \cdot 00$ & $0.50(0 \cdot 10,2 \cdot 49)$ & $1.76(0.92,3.37)$ & $1 \cdot 00$ & $0.49(0.08,3.03)$ \\
\hline $\begin{array}{l}\text { Satisfied with the number of family members living nearby } \\
\quad \text { (yes/very = ref; somewhat/no/not at all), } n(\%)\end{array}$ & $11(34)$ & $159(35)$ & $40(34)$ & $23(27)$ & $154(28)$ & $8(27)$ \\
\hline OR $(95 \% \mathrm{Cl})$ & $0.96(0.51,1.80)$ & $1 \cdot 00$ & $0.96(0.58,1.61)$ & $0.98(0.52,1.85)$ & $1 \cdot 00$ & $0.97(0.41,2.33)$ \\
\hline $\begin{array}{l}\text { Satisfied with the number of friends living nearby } \\
\text { (yes/very = ref; somewhat/no/not at all), } n(\%)\end{array}$ & $11(32)$ & $166(37)$ & $42(37)$ & 17 (19) & $139(25)$ & $11(35)$ \\
\hline OR $(95 \% \mathrm{Cl})$ & $0.82(0.42,1.58)$ & $1 \cdot 00$ & $0.98(0.70,1 \cdot 37)$ & $0.72(0.32,1 \cdot 65)$ & $1 \cdot 00$ & $1.59(0.70,3 \cdot 58)$ \\
\hline Engaging in social activities (no = ref; yes), $n(\%)$ & $8(23)$ & $80(18)$ & $14(12)$ & $28(35)$ & $184(35)$ & $11(36)$ \\
\hline OR $(95 \% \mathrm{Cl})$ & $1 \cdot 35(0.64,2 \cdot 83)$ & $1 \cdot 00$ & $0.61(0.23,1.59)$ & $1 \cdot 01(0.59,1 \cdot 72)$ & $1 \cdot 00$ & $1 \cdot 03(0.44,2 \cdot 40)$ \\
\hline \multicolumn{7}{|l|}{ Block 6: Parental characteristics } \\
\hline Mother's education (illiterate = ref; elementary), $n(\%)$ & $12(40)$ & $167(39)$ & $35(32)$ & $21(26)$ & $177(34)$ & $9(30)$ \\
\hline OR (95\% Cl) & $1 \cdot 11(0 \cdot 47,2 \cdot 61)$ & $1 \cdot 00$ & $0.95(0.52,1 \cdot 74)$ & $0.66(0.37,1 \cdot 19)$ & $1 \cdot 00$ & $1 \cdot 17(0 \cdot 47,2 \cdot 91)$ \\
\hline (illiterate = ref; intermediate and above), $n(\%)$ & $7(26)$ & $106(25)$ & $38(36)$ & $25(33)$ & $156(30)$ & $13(43)$ \\
\hline OR $(95 \% \mathrm{Cl})$ & $1 \cdot 13(0.39,3.30)$ & 1.00 & $1 \cdot 67(1.01,2 \cdot 76)$ & $0.93(0.50 \cdot 1 \cdot 74)$ & 1.00 & $1.90(0.82,4.39)$ \\
\hline Mother works (no = ref; yes), $n(\%)$ & $2(9)$ & $41(10)$ & $25(23)$ & $8(10)$ & $63(12)$ & $7(22)$ \\
\hline OR (95\% Cl) & $0.95(0.21,4.26)$ & $1 \cdot 00$ & $2 \cdot 77(1 \cdot 56,4 \cdot 91)$ & $0.80(0.34,1 \cdot 89)$ & $1 \cdot 00$ & $1.97(0 \cdot 77,5 \cdot 00)$ \\
\hline Father's education (illiterate $=$ ref; elementary), $n(\%)$ & $12(44)$ & $168(43)$ & $36(38)$ & $30(40)$ & $182(39)$ & $9(31)$ \\
\hline OR (95\% Cl) & $0.88(0.30,2 \cdot 56)$ & $1 \cdot 00$ & $0 \cdot 71(0.39,1 \cdot 27)$ & $1 \cdot 53(0.79,2 \cdot 94)$ & $1 \cdot 00$ & $0 \cdot 85(0 \cdot 33,2 \cdot 15)$ \\
\hline (illiterate $=$ ref; intermediate and above), $n(\%)$ & $6(21)$ & $104(27)$ & $22(24)$ & $24(34)$ & $113(24)$ & $10(34)$ \\
\hline OR (95\% Cl) & $0.69(0.25,1.91)$ & 1.00 & $0.71(0.38,1 \cdot 35)$ & $2 \cdot 10(1 \cdot 10,3 \cdot 99)$ & 1.00 & $1.48(0.58,3.78)$ \\
\hline $\begin{array}{l}\text { Father's occupation (own-business } / \text { manager }=\text { ref; } \\
\text { self-employed), } n(\%)\end{array}$ & $10(36)$ & $136(36)$ & $34(38)$ & $27(37)$ & $152(34)$ & $7(25)$ \\
\hline OR $(95 \% \mathrm{Cl})$ & $0.86(0.25,2.93)$ & $1 \cdot 00$ & $0.71(0.36,1 \cdot 41)$ & $2 \cdot 08(0 \cdot 81,5 \cdot 30)$ & $1 \cdot 00$ & $0.58(0.20,1.69)$ \\
\hline
\end{tabular}


was almost double that for girls (see Table 1). LOSEWT were $14.7(95 \%$ CI $6 \cdot 08,35 \cdot 6)$ times more likely to believe thinner is better compared with DONOWT; a similar estimate was observed for satisfaction with weight. However, LOSEWT were $0.22(95 \%$ CI $0.07,0.63)$ times less likely to say girls look beautiful regardless of their weight compared with DONOWT (see Table 1).

Satisfaction with weight, thinner is better and only thin girls are beautiful were the variables retained after adjusting for demographic and health-related variables among boys. Under Model I, the estimates for thinner is better and satisfaction with weight were intensified for GAINWT, whereas they were attenuated for LOSEWT. Furthermore, boys trying to gain weight were $2 \cdot 23(95 \%$ CI $1 \cdot 14,4 \cdot 37)$ times more likely to say that only thin girls are beautiful (see Table 2).

For both boys and girls, care should be taken when interpreting the estimates for satisfaction with weight due to the wide confidence intervals associated with this variable.

\section{Block 2: Self-esteem}

There was evidence for a difference in satisfaction with oneself and achieving like others when comparing LOSEWT and DONOWT. The odds for the aforementioned variables were respectively $2 \cdot 37$ (95\% CI $1 \cdot 38$, $4.06)$ and 0.58 (95\% CI $0.38,0.89)$ for girls; and 2.92 (95\% CI 1.30, 6.57) and $0 \cdot 25$ (95\% CI 0.09, 0.74) for boys. No such evidence was detected for variables in this block when comparing GAINWT and DONOWT (see Table 1).

Satisfaction with oneself was the only variable retained after adjustment for demographic and health-related variables under Model I for girls. For boys, the variables satisfaction with oneself and achieving like others were retained under Model I. The estimates for the adjusted odds were similar to the crude ones (see Table 2).

\section{Block 3: Personal characteristics}

In this block, evidence of a difference among the groups was observed for the variable engaging in a cultural activity but not for the religiosity variables. For girls, the difference was observed in LOSEWT, OR $=1.73(95 \% \mathrm{CI}$ $1 \cdot 14,2 \cdot 64$ ). However, for boys it was observed in GAINWT, OR $=2 \cdot 51(95 \%$ CI $1 \cdot 21,5 \cdot 19)$ (see Table 1$)$.

After adjustment for demographic and health-related variables, no variables in this block were retained under Model I for girls. Among boys, the only variable retained was engaging in a cultural activity with odds of $2 \cdot 17$ (95\% CI $1 \cdot 05,4 \cdot 46)$ when comparing GAINWT and DONOWT (see Table 2).

\section{Block 4: Risky bebaviours}

Smoking cigarettes and argileh were highly associated with each other. In addition, cross-tabulating these two variables with the outcome variable resulted in a sparse table. Therefore, we combined these two variables. The new variable reflected whether an adolescent has ever 
Table 2 Adjusted odds ratios (and corresponding $95 \%$ confidence intervals) under Models I and II* comparing LOSEWT and GAINWT with DONOWT (significant odds with respect to the DONOWT group indicated by italic font): adolescents aged 13-19-years from three underprivileged urban areas of Beirut, Lebanon, 2003

\begin{tabular}{|c|c|c|c|c|c|c|c|c|}
\hline \multirow[b]{3}{*}{ Variable } & \multicolumn{4}{|c|}{ Girls } & \multicolumn{4}{|c|}{ Boys } \\
\hline & \multicolumn{2}{|c|}{ GAINWT } & \multicolumn{2}{|c|}{ LOSEWT } & \multicolumn{2}{|c|}{ GAINWT } & \multicolumn{2}{|c|}{ LOSEWT } \\
\hline & Model I & Model II & Model I & Model II & Model I & Model II & Model I & Model II \\
\hline \multicolumn{9}{|l|}{$\begin{array}{l}\text { Block 1: Attitudes towards thinness } \\
\text { and weight satisfaction }\end{array}$} \\
\hline Thinner is better (no = ref; yes) & $0.15(0.05,0.45)$ & $0.09(0.02,0.37)$ & $2.04(1.06,3.94)$ & $2 \cdot 10(1 \cdot 02,4 \cdot 33)$ & $0.16(0.06,0.42)$ & $0.18(0.07,0.50)$ & $6.99(2.72,17.9)$ & $7 \cdot 77(2 \cdot 50,24 \cdot 1)$ \\
\hline $\begin{array}{l}\text { Satisfied with weight (satisfied/very } \\
\text { satisfied = ref; not/not at all/no } \\
\text { opinion) }\end{array}$ & $17.9(8.06,39 \cdot 7)$ & $30 \cdot 3(13 \cdot 1,70 \cdot 3)$ & $8.47(4.59,15 \cdot 6)$ & $8 \cdot 20(3 \cdot 89,17 \cdot 3)$ & $22 \cdot 9(11 \cdot 5,45 \cdot 4)$ & $33 \cdot 5(16 \cdot 5,68 \cdot 7)$ & $9.53(4.05,22.4)$ & $8.90(3.45,22.9)$ \\
\hline $\begin{array}{l}\text { Only thin girls are beautiful (no = ref; } \\
\text { yes) }\end{array}$ & & & & & $2 \cdot 23(1 \cdot 14,4 \cdot 37)$ & $2 \cdot 23(1 \cdot 13,4 \cdot 40)$ & $1 \cdot 46(0 \cdot 49,4 \cdot 31)$ & $1 \cdot 50(0 \cdot 43,5 \cdot 28)$ \\
\hline \multicolumn{9}{|l|}{ Block 2: Self-esteem } \\
\hline $\begin{array}{l}\text { Satisfied with oneself (agree = ref; } \\
\text { disagree/no opinion) }\end{array}$ & $0.93(0.23,3.79)$ & $0.21(0.02,1 \cdot 82)$ & $2 \cdot 23(1 \cdot 25,3.99)$ & $1 \cdot 38(0 \cdot 59,3 \cdot 23)$ & $1.41(0.63,3 \cdot 16)$ & $0.93(0.29,3.00)$ & $3 \cdot 50(1 \cdot 38,8 \cdot 87)$ & $2 \cdot 35(0 \cdot 43,12 \cdot 9)$ \\
\hline $\begin{array}{l}\text { Can achieve like others (agree = ref; } \\
\text { disagree/no opinion) }\end{array}$ & & & & & $1 \cdot 48(0.92,2 \cdot 38)$ & $2 \cdot 54(1 \cdot 50,4 \cdot 32)$ & $0.26(0.07,0.93)$ & $0.53(0.13,2 \cdot 20)$ \\
\hline \multicolumn{9}{|l|}{ Block 3: Personal characteristics } \\
\hline $\begin{array}{l}\text { Engaged in a cultural activity } \\
\text { (no = ref; yes) }\end{array}$ & & & & & $2 \cdot 17(1 \cdot 05,4 \cdot 46)$ & $2 \cdot 64(1 \cdot 09,6 \cdot 35)$ & $2 \cdot 17(0 \cdot 67,7 \cdot 02)$ & $1.52(0.31,7.36)$ \\
\hline \multicolumn{9}{|l|}{ Block 4: Risky behaviours } \\
\hline $\begin{array}{l}\text { Ever tried cigarettes or argileh } \\
\text { (never = ref; one of them) }\end{array}$ & & $0.29(0.09,0.92)$ & $2 \cdot 80(1 \cdot 64,4 \cdot 79)$ & $2 \cdot 25(1 \cdot 10,4 \cdot 58)$ & & & & \\
\hline (never = ref; both) & $0.29(0.06,1.43)$ & $0.23(0.02,2 \cdot 67)$ & $3 \cdot 77(1 \cdot 98,7 \cdot 19)$ & $2 \cdot 68(0.98,7 \cdot 32)$ & & & & \\
\hline $\begin{array}{l}\text { Violence in the past three months } \\
\text { (no = ref; yes) }\end{array}$ & & & & & $1.50(0.88,2.53)$ & $1.04(0.46,2 \cdot 35)$ & $4.99(2 \cdot 46,10 \cdot 1)$ & $3.75(1.59,8.86)$ \\
\hline $\begin{array}{l}\text { Sibai's physical activity (yes = ref; } \\
\text { no) }\end{array}$ & $1.09(0.41,2.86)$ & $0.64(0.17,2 \cdot 45)$ & $0.50(0.32,0.79)$ & $0.38(0.19,0.75)$ & $0.42(0.26,0.69)$ & $0.43(0.22,0.83)$ & $0 \cdot 48(0 \cdot 19,1 \cdot 19)$ & $0.56(0.18,1 \cdot 72)$ \\
\hline \multicolumn{9}{|l|}{ Block 5: Social support } \\
\hline Membership of a club (no = ref; yes) & & & & & $1 \cdot 37(0 \cdot 88,2 \cdot 13)$ & $1.09(0.55,2 \cdot 14)$ & $3 \cdot 14(1 \cdot 24,7 \cdot 98)$ & $3.56(1 \cdot 08,11 \cdot 7)$ \\
\hline $\begin{array}{l}\text { In need to borrow } 15000 \mathrm{LL} \text {, ask } \\
\text { (family = ref; friends/others/no } \\
\text { one) }\end{array}$ & & & & & $1.88(0.94,3.76)$ & $1 \cdot 79(0.74,4 \cdot 33)$ & $2 \cdot 84(1 \cdot 09,7 \cdot 36)$ & $4.02(0.97,16.6)$ \\
\hline $\begin{array}{l}\text { Personal problem, ask (family = ref; } \\
\text { friends/others/no one) }\end{array}$ & $1 \cdot 39(0 \cdot 36,5 \cdot 37)$ & $2.95(0.74,11 \cdot 8)$ & $1 \cdot 89(1 \cdot 04,3 \cdot 44)$ & $1.94(0.90,4 \cdot 19)$ & & & & \\
\hline $\begin{array}{l}\text { Know people in the neighbourhood } \\
\text { (few/very few = ref; many/most) }\end{array}$ & $2 \cdot 16(0 \cdot 84,5 \cdot 53)$ & $2 \cdot 72(0.92,8.02)$ & $1 \cdot 71(1 \cdot 11,2 \cdot 64)$ & $1.58(0.87,2 \cdot 88)$ & & & & \\
\hline $\begin{array}{l}\text { Block 6: Parental characteristics } \\
\text { Mother works (no = ref; yes) }\end{array}$ & $0.82(0.17,4.08)$ & $0.49(0.09,2 \cdot 75)$ & $2.64(1.42,4.92)$ & $2 \cdot 50(1 \cdot 25,5 \cdot 00)$ & & & & \\
\hline
\end{tabular}

GAINWT, group who were trying to gain weight; DONOWT, group who were doing nothing about their weight; LOSEWT, group who were trying to lose weight.

*Model I estimates are the estimates obtained at the final stage of a stepwise-backward elimination multinomial-logistic regression model for each block of variables controlling for the aforementioned sociodemographic and health variables. Model II estimates are based on the model that includes all the variables retained in the final steps under Model I and controlling for the sociodemographic and health variables. 
tried smoking both cigarettes and argileh, one of them or neither. For GAINWT boys, the odds of smoking one or both were almost three times the odds of smoking in DONOWT. For LOSEWT girls, the odds of smoking one was almost three times the odds in DONOWT and the odds of smoking both was almost four times. Engaging in a fight was statistically significant among boys only; $\mathrm{OR}=1 \cdot 64(95 \% \mathrm{CI} 1 \cdot 03,2 \cdot 60)$ for GAINWT and OR $=$ $5 \cdot 25(95 \%$ CI $2 \cdot 51,11 \cdot 0)$ for LOSEWT (see Table 1$)$.

Just less than half the girls engaged in any kind of sports, compared with about $70 \%$ of the boys. Based on Sibai's classification, there was evidence for a difference in physical activity comparing LOSEWT and DONOWT among girls. LOSEWT were twice as likely to be engaged in physical activity. Similar estimates were obtained for boys in GAINWT and LOSEWT. Using Lowry's classification gave similar estimates, though inflated slightly for girls while deflated slightly for boys. Henceforth, for physical activity we use the classification according to Sibai as we believe it reflects better the practices of this society (see Table 1).

After adjustment for demographic and health-related variables, under Model I physical activity and smoking were retained for girls; whereas physical activity and violence were retained for boys. The adjusted odds were comparable to the crude ones (see Table 2).

\section{Block 5: Social support}

Among boys, comparing LOSEWT and DONOWT, there was evidence of a significant difference for the following variables: membership of a club, turning to no one $v$. a family member when in need of an emergency lift and turning to other than a family member if facing a personal problem. The odds were approximately 3 for all of the aforementioned variables. Compared with DONOWT, GAINWT were about twice as likely to turn to no one $v$. a family member when in need of an emergency lift and to turn to other than a family member if in need to borrow 15 000LL (see Table 1).

The odds of LOSEWT girls turning to other than a family member if facing a personal problem were almost twice those of DONOWT; whereas the odds of knowing many/most people in the neighbourhood was about 1.6 (see Table 1).

The social capital variables retained in Model I after adjustment for demographic and health-related variables were membership of a club and who to turn to when in need to borrow 15 000LL for boys. For girls, the variables retained were who to turn to when facing a personal problem and knowing people in the neighbourhood (see Table 2).

\section{Block 6: Parental characteristics}

No statistical difference was detected among the weightcontrol behaviour groups with regard to father's education or occupation and the mother's education for either gender. However, the mothers of LOSEWT girls were $2 \cdot 77$ (95\% CI $1.56,4.91)$ times more likely to be working and this was statistically significant. This was not the case among boys (see Table 2).

\section{Model II}

Variables in Blocks 1 to 6 that were retained in the final step of the different runs of Model I were included in Model II (see Table 2). The following variables were common for both boys and girls: thinner is better, satisfied with weight/self and physical activity. For girls, the following variables were also included in Model II: ever tried cigarettes/argileh, personal problem, knows people in the neighbourhood and mother works. Also included in Model II for boys were the following additional variables: only thin girls are beautiful, can achieve like others, engaged in a cultural activity, violence in the past three months, membership of a club and who to turn to when in need to borrow 15000 LL. When analysed all together, social capital and cultural capital variables were not significant determinants for weight-control behaviours. Attitudes towards thinness, weight satisfaction and risky behaviours were determinants for boys' and girls' weightcontrol behaviours (see Table 2).

\section{Discussion}

Results of the present research indicate that personal, social and psychological variables, along with other health-risk behaviours, influence weight-control behaviours among adolescents in Beirut. This supports previous research highlighting the complexity of weightcontrol behaviours. However, to our knowledge it is always the case that one subset of these determinants is considered. Our results indicated that determinants vary by weight-control outcome studied (weight loss, weight gain or weight maintenance) and by gender. This extends previous international research.

Demographic variables such as age and education were not significantly different among the three groups. Our results add to the diversity in results related to socioeconomic status. Receiving pocket money, a possible proxy for socio-economic status, was unrelated to weightcontrol behaviour for either gender. So were father's and mother's education and father's occupation. Mother's working status was related to trying to lose weight only among girls.

With respect to health status, those who perceived their health to be worse than others were more likely to be trying to gain weight (data not shown); however, these differences wane when the different blocks of variables are considered. This category of respondents may in fact have a medical condition that resulted in their losing some weight. It may also be a result of perceptions that those who are thinner are less healthy. Traditionally in 
Arab societies, cultural norms placed value on and associated health with plumpness ${ }^{333}$. Although this norm seems to be changing, remnants among parents of our respondents may result in their associating thinness with less 'health'. This is supported by the results of attitudes towards thinness. Those trying to lose weight were more likely to favour thinness. Dissatisfaction with weight was almost equally related to trying to gain or lose weight for both genders. However, when controlling for other variables, those trying to gain weight were most likely to be dissatisfied with their weight. This supports previous literature on the importance of perception of body in behaviours related to weight control ${ }^{(20,22-24)}$.

In support of previous literature ${ }^{(25)}$, self-esteem was related to weight-control behaviour among boys and girls. Interestingly, the two self-esteem items were related in different ways to weight-control behaviour. For both boys and girls, being dissatisfied with oneself was most linked to weight-loss behaviours. For boys, feeling one could not do things as well as others was most linked to gaining weight. However, when controlling for all the blocks, dissatisfaction with oneself was not statistically significant. These results suggest the need for a more careful analysis of the link in research between selfesteem and weight-control behaviours.

With respect to exercise and risky behaviours, girls engaging in exercise were trying to lose weight whereas boys engaging in exercise were trying to gain or lose weight. In addition, supporting the previous literature, smoking cigarettes and argileh was linked to trying to lose weight in girls; whereas for boys, it was linked to trying to gain weight. For boys, engaging in a physical fight in the last three months was linked to trying to lose weight. This link between violence and weight-control behaviours among boys has not been documented previously to our knowledge.

Social capital and cultural capital variables were included in our analysis of determinants of weight-control behaviours. With respect to social capital, there have been contradictory results linking the construct to health ${ }^{(37)}$. Our results support the conclusion that social capital is protective for health with respect to weightcontrol behaviours. Girls and boys who could rely on their families for help were generally more likely to be trying to do nothing about their weight. Boys who were members of clubs were more likely to be trying to lose weight than gain or do nothing. This may be a result of peer pressure to conform to a norm of thinness. We also assessed cultural capital variables ${ }^{(38)}$. In general, cultural capital variables were more relevant to boys trying to lose weight. The same conclusion can be stated for girls for those specific cultural capital variables that were significant in their case. Religiosity was not associated with weight-control behaviours.

In addition, the results described here advance the understanding of determinants of weight-control behaviour specifically among adolescents in Lebanon. Whereas previous research has focused on samples selected from within schools or universities, the adolescents described here are a community sample. Weight-control behaviours were found to differ slightly from previous literature in Lebanon. Fewer respondents reported trying to lose weight, even among girls. In fact, about half as many girl respondents to this survey reported trying to lose weight (20\%) compared with other surveys ${ }^{(7,33,35)}(36-52 \%)$. This may be a result of the younger mean age ( 15 years) of the present sample of respondents as compared with previous studies (17+ years). It may also be a function of different data collection methods. This survey utilized a face-to-face interview with the youth to collect data whereas the others relied on self-report.

The results of the present analysis are subject to several limitations. Importantly, we were unable to include BMI as a confounding variable in this research and therefore do not know if the desire to lose weight, gain weight or do nothing is justified. The survey did not ask respondents' height and weight. Although measured BMI is most accurate, selfreported BMI is used most frequently in research describing determinant weight-control behaviour. Research, however, has suggested that self-reported BMI may not be a valid and reliable measure of actual BMI as people tend to overestimate their height and underestimate their weight ${ }^{(39,40)}$. In Lebanon, previous unpublished research found similar inaccurate results for self-reported height and weight. This may be due to lack of access to regular care, which would be especially important in accurate estimates for adolescents' height and weight. Despite the above justifications, the inability to compare reported desired weight-control behaviours with BMI remains a limitation.

In addition, the survey is cross-sectional and thus relationships must be interpreted as suggested associations, not causal pathways. It is possible that directionality may be reversed and that, for example, trying to lose weight is the determinant of an attitude valuing thinness rather than the reverse. The survey was administered by an interviewer, thus the bias of social desirability cannot be ruled out. Finally, the study was conducted with adolescents aged 13-19 years in three disadvantaged urban areas of Beirut, and so the results are not necessarily generalizable to all adolescents in Lebanon.

The research described herein adds to the international literature on weight-control behaviours among adolescents by providing a comprehensive picture of determinants at various levels of influence and by describing such determinants for adolescents in a developing world setting. Moreover, to our knowledge, the present study is the first one to investigate the impact of social capital and cultural capital variables on adolescents' weight-control behaviours. More research on these specific influences is needed. Results of our study can inform interventions to improve weight-control behaviours among adolescents in urban impoverished areas of Lebanon. 


\section{Acknowledgements}

Sources of funding: Part of this research was completed while M.N.K. was supported by the Hewlett Foundation Research Leave at the Department of Epidemiology and Population Health, Faculty of Health Sciences, American University of Beirut, Beirut, Lebanon. Conflict of interest: The authors declare no conflict of interest. Authorship responsibilities: M.N.K. performed the data analysis and drafted the introduction, methods and results of the manuscript. R.A.A. initiated the manuscript, provided significant advice and contributed to the discussion of the manuscript. Acknowledgments: We would like to thank the CRPH at the American University of Beirut for providing the data for the study. Data used in the current paper were collected as a result of grant support from the Wellcome Trust, the Ford Foundation and the Mellon Foundation.

\section{References}

1. United Nations (2008) Millennium Development Goals. End Poverty 2015: Make it happen. http://www.un.org/ millenniumgoals/ (accessed January 2009).

2. World Health Organization (2003) Obesity and overweight fact sheet. http://www.who.int/dietphysicalactivity/media/ en/gsfs_obesity.pdf (accessed January 2009).

3. World Health Organization (2000) Obesity: Preventing and Managing the Global Epidemic. WHO Technical Report Series no. 894. Geneva: WHO; available at http://www.iotf. org/popout.asp?linkto $=$ http://whqlibdoc. who.int/trs/WHO_ TRS_894.pdf

4. de Onis M, Blössner M, Borghi E, Frongillo EA \& Morris R (2004) Measurement and standardization protocols for anthropometry used in the construction of a new international growth reference. Food Nutr Bull 25, Suppl., S27-S36.

5. Caulfield LE, de Onis M, Blössner M \& Black RE (2004) Undernutrition as an underlying cause of child deaths associated with diarrhea, pneumonia, malaria, and measles. Am J Clin Nutr 80, 193-198.

6. Khatib O (2004) Noncommunicable diseases: risk factors and regional strategies for prevention and care. East Mediterr Health J 10, 778-788.

7. Sibai AM, Hwalla N, Adra N \& Rahal B (2003) Prevalence and covariates of obesity in Lebanon: findings from the first epidemiological study. Obes Res 11, 1353-1361.

8. Djazayery A (2004) Regional overview of maternal and child malnutrition: trends, interventions and outcomes. East Mediterr Health J 10, 731-736.

9. Delisle H, Chandra-Mouli V \& De Benoist B (2001) Should Adolescents be Specifically Targeted for Nutrition in Developing Countries? To Address which Problems, and How? Geneva: WHO; available at http://www.who.int/ child-adolescent-health/New_Publications/NUTRITION/ Adolescent_nutrition_paper.pdf (accessed October 2005).

10. Stice E \& Shaw HE (2002) Role of body dissatisfaction in the onset and maintenance of eating pathology: a synthesis of research findings. J Psychosom Res 53, 985-993.

11. Kelder SH, Perry CL, Klepp KI \& Lytle LL (1994) Longitudinal tracking of adolescent smoking, physical activity, and food choice behaviours. Am J Public Health 84, $1121-1126$.

12. Sweeting H, Anderson A \& West P (1994) Socio-demographic correlates of dietary habits in mid to late adolescence. Eur J Clin Nutr 48, 736-748.
13. Patton GC, Selzer R, Coffey C, Carlin JB \& Wolfe R (1999) Onset of adolescent eating disorders: population based cohort study over 3 years. BMJ 318, 765-768.

14. Paxton SJ (2002) Body dissatisfaction and disordered eating. J Psychosom Res 53, 961-962.

15. Kilpatrick M, Ohannessian C \& Bartholomew JB (1999) Adolescent weight management and perceptions: an analysis of the National Longitudinal Study of Adolescent Health. J Sch Health 69, 148-1152.

16. Adams K, Sargent RG, Thompson SH, Richter D, Corwin SJ \& Rogan TJ (2000) A study of body weight concerns and weight control practices of 4 th and 7 th grade adolescents. Ethn Health 5, 79-94.

17. Neumark-Sztainer D \& Hannan PJ (2000) Weight-related behaviours among adolescent girls and boys: results from a national survey. Arch Pediatr Adolesc Med 154, 569-577.

18. Lowry R, Galuska DA, Fulton JE, Wechsler H \& Kann L (2002) Weight management goals and practices among US high school students: associations with physical activity, diet, and smoking. J Adolesc Health 31, 133-144.

19. Lowry R, Galuska DA, Fulton JE, Burgeson CR \& Kann L (2005) Weight management goals and use of exercise for weight control among US high school students, 1991-2001. $J$ Adolesc Health 36, 320-326.

20. Neumark-Sztainer D, Story M, Resnick MD \& Blum RW (1998) Lessons learned about adolescent nutrition from the Minnesota Adolescent Health Survey. J Am Diet Assoc 98, 1449-1456.

21. Crocker P, Kowalski N, Kowalski K, Chad K, Humbert L \& Forrester S (2001) Smoking behaviour and dietary restraint in young adolescent women: the role of physical selfperceptions. Can J Public Health 92, 428-432.

22. Ackard DM, Croll JK \& Kearney-Cooke A (2002) Dieting frequency among college females: association with disordered eating, body image, and related psychological problems. J Psychosom Res 52, 129-136.

23. Franko DL \& Striegel-Moore RH (2002) The role of body dissatisfaction as a risk factor for depression in adolescent girls: are the differences black and white? J Psychosom Res 53, 975-983.

24. Crow S, Eisenberg ME, Story M \& Neumark-Sztainer D (2006) Psychosocial and behavioural correlates of dieting among overweight and non-overweight adolescents. $J$ Adolesc Health 38, 569-574.

25. Viner RM, Haines MM, Taylor SJ, Head J, Booy R \& Stansfeld S (2006) Body mass, weight control behaviours, weight perception and emotional well being in a multiethnic sample of early adolescents. Int J Obes (Lond) 30, $1514-1521$.

26. Dunkley TL, Wertheim EH \& Paxton SJ (2001) Examination of a model of multiple sociocultural influences on adolescent girls' body dissatisfaction and dietary restraint. Adolescence 36, 265-279.

27. Field AE, Camargo Jr CA, Taylor CB, Berkey CS, Roberts SB \& Colditz GA (2001) Peer, parent, and media influences on the development of weight concerns and frequent dieting among preadolescent and adolescent girls and boys. Pediatrics 107, 54-60.

28. Neumark-Sztainer D, Story M, Toporoff E, Himes JH, Resnick MD \& Blum RW (1997) Covariations of eating behaviours with other health-related behaviours among adolescents. J Adolesc Health 20, 450-458.

29. Barker M, Robinson S, Wilman C \& Barker DJ (2000) Behaviour, body composition and diet in adolescent girls. Appetite 35, 161-170.

30. Rafiroiu C, Sargent RG, Parra-Medina D, Drane WJ \& Valois RF (2003) Covariations of adolescent weight-control, health-risk and health-promoting behaviours. Am J Health Behav 27, 3-14. 
31. Fulkerson JA \& French SA (2003) Cigarette smoking for weight loss or control among adolescents: gender and racial/ethnic differences. J Adolesc Health 32, 306-313.

32. Austin SB \& Gortmaker SL (2001) Dieting and smoking initiation in early adolescent girls and boys: a prospective study. Am J Public Health 91, 446-450.

33. Afifi-Soweid RA, Najem Kteily MB \& Shediac-Rizkallah MC (2002) Preoccupation with weight and disordered eating behaviours of entering students at a university in Lebanon. Int J Eat Disord 32, 52-57.

34. Khawaja M \& Afifi-Soweid RA (2004) Images of body weight among young men and women: evidence from Beirut, Lebanon. J Epidemiol Community Health 58, 352-353.

35. Tamim H, Dumit N, Terro A, Al-Hourany R, Sinno D, Seif F, Steitieh S \& Musharrafieh U (2004) Weight control measures among university students in a developing country: a cultural association or a risk behaviour. J Am Coll Nutr $\mathbf{2 3}$ 391-396.

36. Lowry R, Galuska DA, Fulton JE, Wechsler H, Kann L \& Collins JL (2000) Physical activity, food choice, and weight management goals and practices among US college students. Am J Prev Med 18, 18-27.

37. Kreuter MW \& Lezin N (2002) Social capital theory: implications for community-based health promotion. In Emerging Theories in Health Promotion Practice and Research: Strategies for Improving Public Health, pp. 228-254 [RJ DiClemente, RA Crosby and MC Kegler, editors]. San Francisco, CA: Jossey-Bass Wiley.

38. Khawaja M \& Mowafi M (2006) Cultural capital and selfrated health in low income women: evidence from the Urban Health Study, Beirut, Lebanon. J Urban Health 83, 444-458.

39. Flood V, Webb K, Lazarus R \& Pang G (2000) Use of selfreport to monitor overweight and obesity in populations: some issues for consideration. Aust N ZJ Public Health 24, 96-99.

40. John U, Hanke M, Grothues J \& Thyrian JR (2006) Validity of overweight and obesity in a nation based on self-report versus measurement device data. Eur J Clin Nutr 60, 372-377. 